\title{
Resultado de pruebas de parche en pacientes con sospecha de dermatitis alérgica de contacto en Medellín, 2006-2013
}

Patch test results in patients with allergic contact dermatitis in Medellin, Colombia, 2006-2013

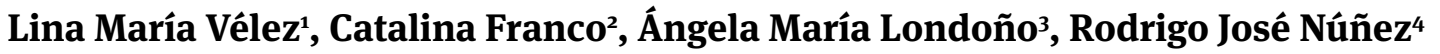 \\ 1. Médica dermatóloga, Universidad Pontificia Bolivariana, Medellín, Colombia. \\ 2. Médica, Universidad Pontificia Bolivariana, Medellín, Colombia. \\ 3. Médica dermatologa, MSc Epidemiologia, docente Universidad CES. \\ 4. Médico dermatólogo, Clínica Universidad Pontificia Bolivariana; docente, Universidad Pontificia Bolivariana, Medellín, Colombia.
}

\section{RESUMEN}

INTRODUCCIón. La dermatitis alérgica de contacto es una reacción inflamatoria de hipersensibilidad tardía a agentes exógenos. En el ambiente existen alrededor de seis millones de sustancias químicas, muchas de las cuales pueden causar sensibilización en las personas. Hasta el momento, solo existe una publicación que refleja el comportamiento de esta entidad en Colombia, la cual resulta insuficiente para hacer predicciones y modificar conductas de riesgo.

OBJETivos. Determinar los principales alérgenos implicados en la dermatitis alérgica de contacto en los pacientes evaluados en dos centros médicos de Medellín.

MATERIALES Y MÉTODOS. Se llevó a cabo un estudio observacional descriptivo, retrospectivo y prospectivo, entre enero de 2006 y diciembre de 2013 de pacientes con hallazgos clínicos sugestivos de dermatitis de contacto, remitidos para pruebas de parche en dos centros médicos de Medellín.

RESUltados. Se recolectaron datos de 1.349 pacientes, $77 \%$ mujeres, y la media

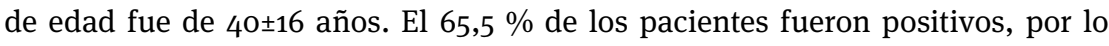
menos, a un alérgeno. Los alérgenos más comunes fueron: sulfato de níquel (35,6 $\%$ ), cloruro de cobalto (16,2 \%), mertiolate (thimerosal) (12,7 \%), bálsamo del Perú $(8,5 \%)$ y dicromato de potasio $(6 \%)$. Las localizaciones más frecuentes de las lesiones fueron la cara (43\%) y las manos (32,1\%).

Conclusiones. La dermatitis alérgica de contacto es un problema frecuente en la consulta dermatológica, y la prueba de parche es una estrategia útil y costoefectiva. Este estudio es la publicación con mayor número de pacientes en Colombia y una de las más grandes en Latinoamérica, y refleja el comportamiento de los principales alérgenos. A pesar de las estrategias internacionales de control del níquel, en Colombia persisten altas tasas de sensibilización.

PALABRAS CLAVE: dermatitis alérgica de contacto, pruebas de parche.

\section{SUMMARY}

INTRODUCTION: Allergic contact dermatitis is an inflammatory reaction of delayed hypersensitivity to exogenous agents. It is estimated that there are about six million
Correspondencia:

Lina María Vélez

Email:

linavelezp@gmail.com

Recibido: 13 de enero de 2016 Aceptado: 18 de abril de 2016

No se reportan conflictos de interés. 
chemicals in the environment, of which approximately 2,800 may cause sensitization in people. So far there is only one publication that reflects the behavior of this entity in Colombia, which is insufficient to make predictions and modify risk factors. OBJECTIVES: To determine the major allergens involved in allergic contact dermatitis in patients evaluated at to two medical centers in the city of Medellín.

METHODS: A descriptive, retrospective and prospective observational study was conducted between 2006 and 2013 in patients with clinical findings suggestive of contact dermatitis, referred for patch tests to two medical centers in Medellín.

RESULTS: Data from 1,349 patients was collected: the mean age was $40 \pm 16$ years, $77 \%$ female, of which $65.5 \%$ had at least one positive allergen. The most common allergens were: nickel sulfate, $35.6 \%$, cobalt chloride, $16.2 \%$, thimerosal, $12.7 \%$, the balm of Perú, 8.5\%, and potassium dichromate, $6 \%$. Lesions were mainly located in the face (43\%), followed by the hands $(32.1 \%)$.

Conclusions: Allergic contact dermatitis is a common problem seen by dermatologists. Patch testing is a useful and cost-effective strategy in the diagnostic and therapeutic approach. This study is the largest published in Colombia and one of the largest in Latin America, reflecting the behavior of major allergens. Despite international control strategies for nickel, rates are much higher in Colombia than those reported in the United States and Europe.

KEY WORDS: Allergic contact dermatitis, patch test.

\section{INTRODUCCIÓN}

La dermatitis alérgica de contacto es una dermatosis frecuente y representa el $10 \%$ de las consultas a dermatología general y es aún más relevante en la práctica de dermatología ocupacional; es responsable, junto con la dermatitis irritativa de contacto, de aproximadamente el $90 \%$ de las dermatosis profesionales ${ }^{1,2}$.

Clínicamente, se caracteriza por la presencia de lesiones eccematosas, generalmente pruriginosas, que aparecen entre 24 y 72 horas después de haber entrado en contacto con el alérgeno. En procesos más crónicos, se evidencia 'liquenificación' y descamación con persistencia del prurito ${ }^{3,4}$. Generalmente, tienen cursos crónicos y recidivantes, lo que implica gran compromiso de la calidad de vida de los pacientes y alteración de su desempeño laboral ${ }^{5,6}$.

Se estima que en el ambiente existen alrededor de seis millones de sustancias químicas, de las cuales 2.80o pueden causar sensibilización en las personas ${ }^{7}$.

La prueba de parche se introdujo en la práctica médica hace más de un siglo y, desde entonces, ha sido utilizada tanto en investigación como en la consulta dermatológica y alergológica ${ }^{8}$. Es el método diagnóstico de elección en esta entidad, con una sensibilidad entre el 70 y el $80 \% \%^{9,10}$. Las pruebas de parche implican la aplicación de sustancias específicas de contacto, en condiciones controladas, directamente sobre la piel del paciente, las cuales causan una reacción local en personas previamente sensibilizadas ${ }^{11}$. Las sustancia de contacto que se utilizan han sido determinadas por el International Contact Dermatitis Research Group (ICDRG) y se consideran las responsables más frecuentes de la entidad ${ }^{12}$.

En los últimos años se ha demostrado que la vigilancia clínica y el análisis sistemático de las pruebas de parche son un requisito para la identificación de los alérgenos y la determinación de la prevalencia de la dermatitis alérgica de contacto ${ }^{13}$; esto ha llevado a que la mayoría de las ciudades de Europa y Norteamérica hagan seguimiento e implementación de medidas preventivas en grupos de riesgo expuestos a diferentes sustancias ${ }^{14}$.

En Medellín, hasta el momento solo se ha llevado a cabo un estudio en el que se evaluó la prevalencia de la sensibilización a diferentes sustancias de contacto ${ }^{15}$; sin embargo, un solo estudio resulta insuficiente para hacer cambios e implementar estrategias de prevención que causen un impacto en la calidad de vida de estos pacientes.

\section{MATERIALES Y MÉTODOS}

Se llevó a cabo un estudio observacional, descriptivo, retrospectivo y prospectivo. 
Previa aprobación del proyecto por parte del Comité de Ética de la Universidad Pontificia Bolivariana, se tomó la información de los pacientes por medio del acceso a la historia clínica electrónica de la Clínica Universitaria Bolivariana y a la historia clínica de una consulta de dermatología de Medellín. Se incluyeron todos aquellos pacientes a quienes se les practicaron pruebas de parche entre el $1^{\circ}$ de enero de 2006 y el 31 de diciembre de 2013. Inicialmente, se utilizó la batería europea estándar, la cual incluye 28 haptenos (Chomotechniq ${ }^{\circledR}$ con cámaras de Finn ${ }^{\circledR}$ ) y, a partir del 2012, se incluyeron además el mertiolate (thimerosal) y el lauril sulfato, para un total de 30 haptenos (TABLA 1). A todos los pacientes se les hizo la primera lectura a las 48 horas y la segunda lectura a las 96 horas, de acuerdo con lo establecido por las guías del ICDRG. Todos estos pacientes tenían, entre los diagnósticos presuntivos, la dermatitis alérgica de contacto.

Las reacciones se clasificaron según el eritema, la infiltración o la formación de ampollas que se producen en el sitio de aplicación del alérgeno; una cruz para la presencia de eritema; dos cruces para la formación de eritema, edema e inicio de pápulas o vesículas, y tres cruces cuando había formación de vesículas coalescentes o ampollas. Con una cruz se consideró la prueba positiva.

En los centros de práctica evaluados, no se practicaron pruebas de parche en pacientes embarazadas, con exposición reciente a radiación ultravioleta, con lesiones activas en la espalda o con el antecedente de haber recibido esteroides o inmunosupresores sistémicos 15 días antes de las pruebas de parche, o esteroides tópicos una semana antes.

Todas las pruebas de parche fueron leídas e interpretadas por dos dermatólogos con formación y experiencia en el tema.

Se tuvieron en cuenta aspectos sociodemográficos como sexo, edad y ocupación, y clínicos, como tiempo de evolución y localización de las lesiones, y el resultado a las 96 horas de las pruebas de parche.

\section{Análisis de los datos}

Toda la información fue recolectada y analizada mediante el programa SPSS ${ }^{\circledR}$, versión 19. Se hizo una descripción estadística de la muestra según las variables consideradas en el estudio.

Para las variables cuantitativas se calcularon las medidas de tendencia central y dispersión (media, mediana, desviación estándar y percentiles). Para las variables cualitativas se construyeron tablas con frecuencias absolutas y relativas.

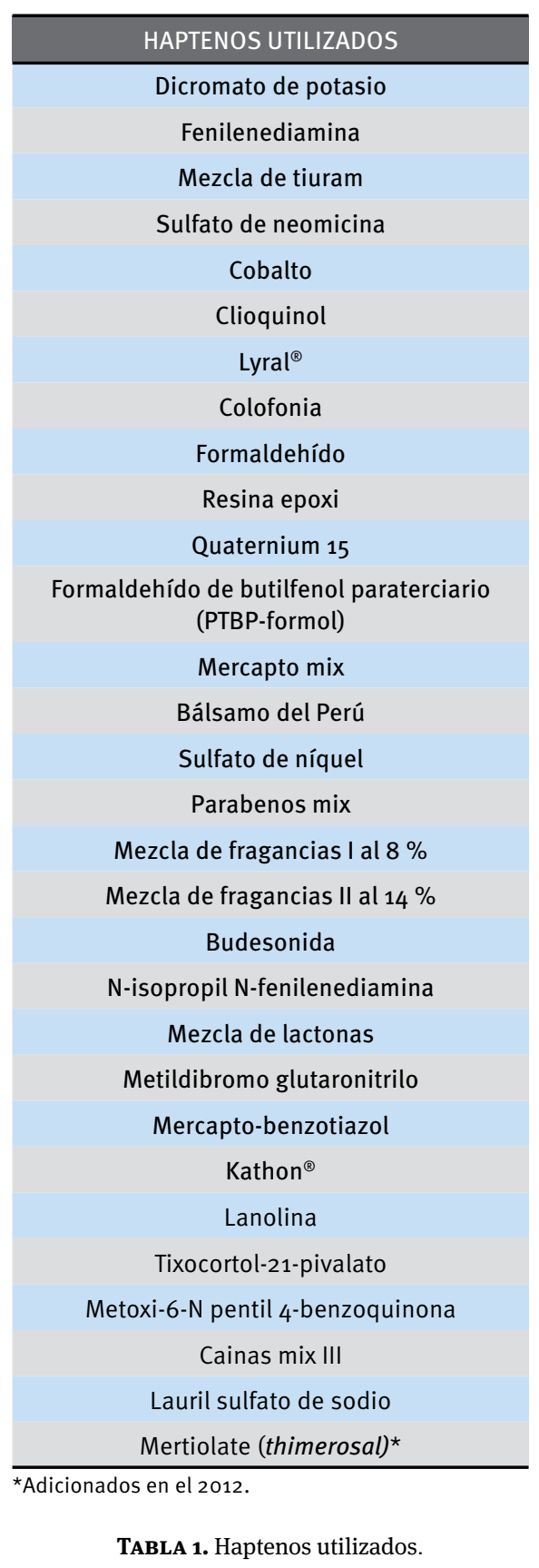

\section{RESULTADOS}

Se recolectaron los datos de 1.349 pacientes a quienes se les practicó la prueba de parche durante el período de estudio. De estos, $77 \%$ eran de sexo femenino $(\mathrm{n}=1.040)$. El rango de edad fue de 6 a 94 años (media, 40,8 $\pm 16,3)$. El 6,2 \% $(n=83)$ de los pacientes eran me- 


\begin{tabular}{|c|c|c|}
\hline & $\mathrm{n}$ & $\%$ \\
\hline \multicolumn{3}{|l|}{ SEXO } \\
\hline Hombres & 309 & 22,9 \\
\hline Mujeres & 1.040 & 77,1 \\
\hline \multicolumn{3}{|l|}{ Edad (años) } \\
\hline$\ll 18$ & 83 & 6,2 \\
\hline 19 a 40 & 603 & 44,7 \\
\hline 41 a 60 & 477 & 35,4 \\
\hline$>60$ & 171 & 12,7 \\
\hline \multicolumn{3}{|l|}{ OCUPACIÓN } \\
\hline Amas de casa & 256 & 19 \\
\hline Salud & 95 & 7 \\
\hline Textiles & 26 & 2 \\
\hline Construcción & 17 & 1,1 \\
\hline Otros & 931 & 69 \\
\hline \multicolumn{3}{|c|}{ LOCALIZACIÓN DE LAS LESIONES } \\
\hline Cara & 581 & 43 \\
\hline Manos & 434 & 32,1 \\
\hline Generalizado & 263 & 19,5 \\
\hline Pies & 107 & 7,9 \\
\hline Piernas & 101 & 7,5 \\
\hline Cuero cabelludo & 41 & 3 \\
\hline \multicolumn{3}{|c|}{ TIEMPO DE EVOLUCIÓN (MESES) } \\
\hline$\ll 6$ & 381 & 28 \\
\hline 6 a 24 & 538 & 40 \\
\hline 124 & 384 & 28,5 \\
\hline
\end{tabular}

TABLA 2. Características demográficas de la población ( $\mathrm{N}=1.349)$

nores de 18 años. En el grupo de adultos, el mayor porcentaje $(44,7 \%)$ se encontró en las personas entre 19 y 40 años, seguido por el grupo entre 41 a 60 años $(35,4 \%)$ y, por último, el grupo de mayores de 60 años $(12,7 \%)$.

\section{(TABLA 2) (FIGURA 1).}

Los alérgenos más frecuentes fueron: el sulfato de níquel $(n=481,35,6 \%)$, el cloruro de cobalto $(n=219,16,2 \%)$, el mertiolate $(\mathrm{n}=172,12,7 \%)$, el bálsamo del Perú $(\mathrm{n}=115$, $8,5 \%)$ y el dicromato de potasio ( $\mathrm{n}=82,6 \%$ ) (TABLA 3).

La población pediátrica, correspondiente al 6,2 \%, mostró sensibilización al sulfato de níquel como primer alérgeno $(\mathrm{n}=35,31 \%)$, seguido por el cloruro de cobalto $(n=20,17 \%)$, el mertiolate $(n=13,11 \%)$ y el dicromato de potasio ( $\mathrm{n}=4,3,5 \%)$ (TABLA 4).

\begin{tabular}{|lll}
\hline & $\mathrm{n}$ & $\%$ \\
\hline Sulfato de níquel & 481 & \\
\hline Cloruro de cobalto & 219 & 16,2 \\
\hline Mertiolate & 172 & 12,7 \\
\hline Bálsamo del Perú & 115 & 8,5 \\
\hline Dicromato de potasio & 82 & 6 \\
\hline Sulfato de neomicina 20\% & 78 & 5,7 \\
\hline Mezcla de fragancias al 14\% & 61 & 4,5 \\
\hline Parafenilendiamina al 0,1 \% & 53 & 3,9 \\
\hline Quaternium 15 al 0,1 \% & 41 & 3 \\
\hline Mezcla de fragancias al 8\% & 40 & 3 \\
\hline
\end{tabular}

TABLA 3. Alérgenos más frecuentes.

\begin{tabular}{lll}
\hline & $n$ & $\%$ \\
\hline Sulfato de níquel & 35 & 31 \\
\hline Cloruro de cobalto & 20 & 17 \\
\hline Timerosal & 13 & 11 \\
\hline Dicromato de potasio & 4 & 3.5 \\
\hline
\end{tabular}

TABLA 4. Alérgenos más frecuentes en la infancia.

De los 1.349 pacientes, el 65,5\% ( $\mathrm{n}=883)$ era sensible, por lo menos, a un alérgeno; el 30,9\% $(\mathrm{n}=418)$, a ninguno, y en el 3,5\% $(n=48)$ restante no se pudo obtener una historia clínica completa ni información del resultado de la prueba.

Las pruebas fueron positivas para un solo alérgeno en $31 \%(\mathrm{n}=422)$ de los casos; para dos alérgenos, en el 19,5\% $(n=263)$; para tres alérgenos, en el 10,4 \% $(\mathrm{n}=140)$, y el valor máximo por pacientes fue de cuatro de alérgenos positivos en el 4,4\% $(\mathrm{n}=60)$ de los casos.

Las lesiones estuvieron localizadas con mayor frecuencia en la cara en 581 (43\%) pacientes, seguida por las manos en $424(32,1 \%) \mathrm{y}$, en tercer lugar, las lesiones generalizadas en $263(19,5 \%)$. Las localizaciones menos frecuentes fueron los pies en 107 (7,9\%) pacientes; las piernas, en 101 (7,5\%), y el cuero cabelludo en 41 (3\%).

La ocupación más frecuente fue la de ama de casa $(\mathrm{n}=256,19 \%)$, seguida por trabajador del área de la salud $(\mathrm{n}=95,7 \%)$ y trabajador del área textil $(\mathrm{n}=26,2 \%)$; hubo poca representación de otros oficios, como trabajador agrícola y ganadero $(\mathrm{n}=15,1 \%)$, sector de la construcción $(n=17,1,3 \%)$, peluquería $(n=13,1 \%)$, manipulador de alimentos $(\mathrm{n}=14,1 \%)$ y mecánico $(\mathrm{n}=12,0,9 \%)$. El $66 \%(n=890)$ de los pacientes se clasificó en el grupo 


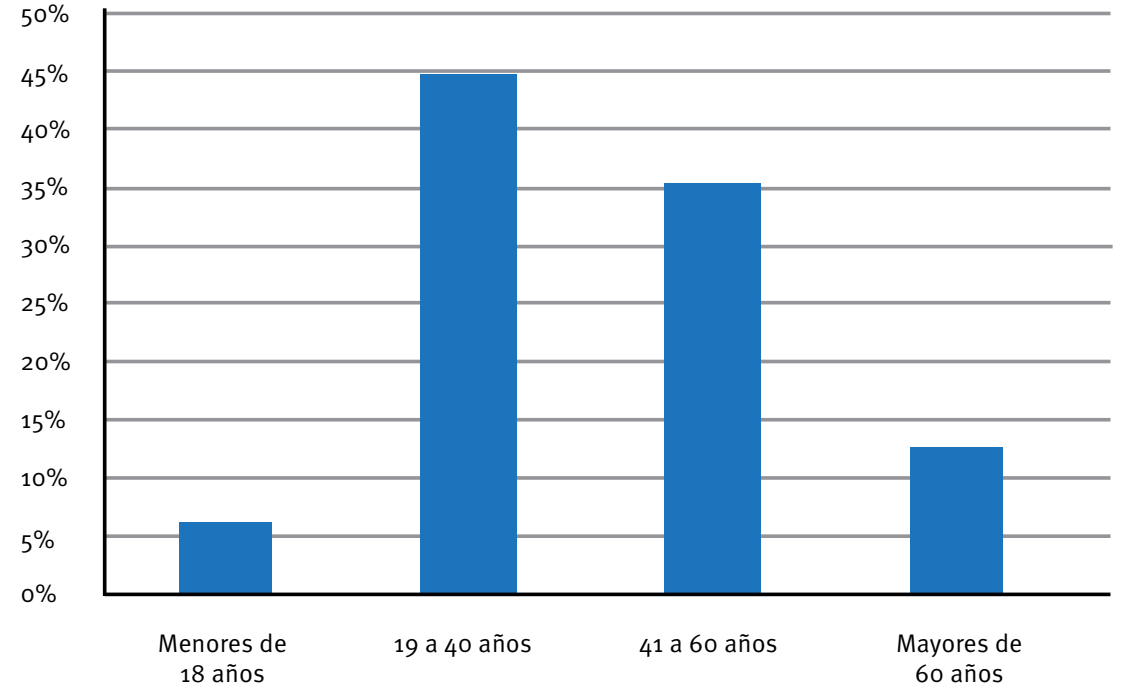

FIGURA 1. Distribución de la frecuencia por grupos de edad. denominado "otros", en el cual se incluían en su mayoría trabajos de oficina.

La evolución de la lesiones fue de menos de seis meses en $28 \%(n=381)$ de los pacientes, de seis meses a dos años en $40 \%(n=539)$ y de más de dos años en $28,5 \%(n=384)$.

\section{DISCUSIÓN}

En el presente estudio se recolectaron datos de 1.349 pacientes en un periodo de ocho años, una muestra mucho mayor a lo reportado en otros estudios de nuestro país y una de las más grandes en Latinoamérica, hasta el momento ${ }^{15-17}$.

Los cinco principales alérgenos fueron: sulfato de níquel (35,6\%), cloruro de cobalto (16,2\%), mertiolate $(12,7 \%)$, bálsamo del Perú $(8,5 \%)$ y dicromato de potasio (6\%), lo cual presenta algunas variaciones con respecto al estudio que se llevó a cabo del 2000 al 2005 en esta ciudad ${ }^{15}$.

El sulfato de níquel continúa siendo el principal alérgeno en nuestra población y su porcentaje no ha disminuido en el tiempo, a pesar de las estrategias de control sobre los productos que contienen este elemento a nivel mundial, implementadas en el 2001. Estas prohíben la venta y la comercialización de sustancias que estén en contacto directo y prolongado con la piel, y que tengan una liberación de níquel superior a $0,5 \mu \mathrm{g}$ por $\mathrm{cm}^{2}{ }^{18}$. El porcentaje de sensibilización a este alérgeno en nuestro medio, duplica los valores encontrados en los Estados Unidos y Europa ${ }^{14,19}$.

El cloruro de cobalto fue el segundo alérgeno en frecuencia; se sabe que se relaciona estrechamente con la sensibilización al níquel debido a que se encuentra como una impureza en accesorios y joyas de fantasía de este metal.

El tercer alérgeno en frecuencia implicado en las pruebas de parches positivas, fue el mertiolate. Esta sustancia que se utiliza como antiséptico o conservante de diferentes medicamentos, cosméticos o vacunas, se agregó a las pruebas de parche a partir del 2012 y llama la atención que en corto tiempo haya logrado un porcentaje significativo en el estudio. Sin embargo, aunque se ha mencionado en muchas series, hasta el momento todavía se cuestiona su relevancia clínica ${ }^{20}$.

El bálsamo del Perú también estuvo presente entre los alérgenos más frecuentes en el estudio llevado a cabo entre el 2000 y 2005 en Medellín ${ }^{15}$, con una ligera disminución en el porcentaje de sensibilización. Los productos químicos encontrados en el bálsamo del Perú son comunes en especias y aromatizantes, productos cosméticos, fragancias y medicinas. Es uno de los alérgenos 
más frecuentes en los Estados Unidos y Europa y puede resultar difícil evitar completamente el contacto con él ${ }^{21}$.

El dicromato de potasio fue el quinto alérgeno en frecuencia, presentándose en $6 \%$ de los casos. Es una de las causas más frecuentes de la dermatitis alérgica de contacto en hombres y su exposición se presenta principalmente en trabajadores de la construcción que tienen contacto con cemento húmedo. En el presente estudio, fue el tercer alérgeno en frecuencia en el grupo de los hombres ( $n=40,13,6 \%)$, superado solamente por el sulfato de níquel $(\mathrm{n}=52,17 \%)$ y el cloruro de cobalto $(n=45,15 \%)$. En este estudio, el dicromato de potasio fue responsable del $22 \%$ de las sensibilizaciones en los trabajadores de la construcción, grupo que fue el más representativo para este alérgeno.

Similar a lo reportado en la literatura científica nacional e internacional, la mayoría de los pacientes fueron mujeres, $77 \%(2,14,15)$. Esto se puede relacionar con el hecho de que las mujeres tienden a consultar con mayor frecuencia y en forma más temprana que los hombres ante cualquier enfermedad en la piel. Esta alta prevalencia también se explica porque las mujeres se sensibilizan al níquel con mayor frecuencia y en forma más temprana que los hombres ${ }^{22}$, y este fue el alérgeno positivo encontrado con mayor frecuencia.

Tanto en el estudio realizado entre el 2000 y el 2005 como en el actual, la población pediátrica tuvo una representación importante $(6,2 \%)$, superior a lo reportado en la literatura científica internacional ${ }^{23}$. A todos los pacientes de este grupo se les practicó la prueba de parche con las mismas concentraciones que a los adultos, de acuerdo con lo sugerido en recientes publicaciones ${ }^{24}$.

Los alérgenos más frecuentes en este grupo de pacientes fueron: sulfato de níquel ( $\mathrm{n}=35,31 \%)$, cloruro de cobalto $(n=20,17 \%)$, mertiolate $(n=13,11 \%)$ y dicromato de potasio $(n=4,3,5 \%)$. Los tres primeros alérgenos son similares a lo reportado en la literatura internacional ${ }^{24,25}$. El sulfato de níquel es el más frecuente en la mayoría de las series. En otros reportes son importantes el sulfato de neomicina y la mezcla de fragancias.

La localización más frecuente de las lesiones fue la cara, contrario a lo que reporta la literatura científica mundial, en la cual el sitio más frecuente son las manos. Esto se puede explicar, en parte, porque la mayor parte de los pacientes incluidos tenían acceso a una práctica dermatológica privada y la sensibilización no tenía origen ocupacional. En la dermatitis alérgica e irritativa de contacto de origen profesional, las manos son la parte del cuerpo más comprometida ${ }^{17}$. En Colombia se presenta un subdiagnóstico y un subregistro importante de las dermatosis profesionales ${ }^{26}$.
Las limitaciones de este estudio son los propios de un estudio descriptivo retrospectivo y prospectivo, entre las cuales se encuentran la falta de seguimiento clínico para corroborar la relevancia de la sustancia de contacto; además, se presentó un sesgo de selección dado que muchos pacientes accedieron al servicio de forma particular y, por lo tanto, no son una muestra representativa, ni revelan el comportamiento de las dermatosis profesionales en nuestro medio.

En el único estudio previo a este en Medellín, se demostró que las sustancias de contacto más frecuentes fueron el sulfato de níquel (36,8 \%) y el cloruro de cobalto $(19,1 \%)$, lo cual es coherente con lo encontrado en el presente estudio. Se demostró, también, una incidencia de dermatitis alérgica de contacto en la edad pediátrica superior a la reportada a nivel mundial ${ }^{15}$.

Consideramos que tener dos estudios en nuestro país, con un aumento del número de pacientes evaluados, favorece la implementación de medidas de prevención, especialmente las relacionadas con el control industrial del níquel.

\section{CONCLUSIONES}

La dermatitis alérgica de contacto es un problema frecuente en la consulta dermatológica, y la prueba de parche es una estrategia útil y costo-efectiva en la aproximación diagnóstica y la terapéutica. Este estudio es la publicación con mayor número de pacientes en Colombia y una de las más grandes en Latinoamérica, la cual refleja el comportamiento de los principales alérgenos. Hasta el momento se tienen múltiples reportes sobre el comportamiento de esta entidad en Estados Unidos y Europa; sin embargo, la información en Latinoamérica es escasa.

Los resultados de este estudio son comparables con los de la literatura científica internacional, excepto en los altos porcentajes de sensibilización al níquel, que duplican los reportados en otras series. Esto, probablemente, se debe a la falta de implementación de estrategias de control.

\section{REFERENCIAS}

1. Ale IS, Maibach HA. Diagnostic approach in allergic and irritant contact dermatitis. Expert Rev Clin Immunol. 2010;6:291-310.

2. Nelson JL, Mowad CM. Allergic contact dermatitis: Patch testing beyond the TRUE test. J Clin Aesthet Dermatol. 2010;3:36-41.

3. Golden S, Shaw T. Hand dermatitis: Review of clinical features and treatment options. Semin Cutan Med Surg. 2013;32:147-57. 
4. Johansen J, Menne T, Christophersen J, Kaaber K, Veien N. Changes in the pattern of sensitization to common contact allergens in Denmark between 1985-1986, with a special view to the effect of preventive strategies. $\mathrm{Br} J$ Dermatol. 2000;142:490-5.

5. Angelini G, Meneghini CL. Patch tests: Advantages and undesirable effects. J Ital Dermatol Venereol. 1986;121:209-14.

6. Wolf R, Orion E, Ruocco E, Baroni A, Ruocco V. Contact dermatitis: Facts and controversies. Clin Dermatol. 2013;31:467-78.

7. Diepgen TL, Coenraads PJ. Sensitivity, specificity and positive predictive value of patch testing: The more you test, the more you get? ESCD Working Party on Epidemiology. Contact Dermatitis. 2000;42:315-7.

8. Wolf R, Orion E, Ruocco E, Baroni A et al. Patch testing: Facts and controversies. Clin Dermatol. 2013;31:479-86.

9. Corazza M, Borghi A, Mantovani L, Virgili A. Analysis of patch test referrals: Influence of appropriateness of referrals on sensitization rate. Contact Dermatitis. 2011;66:95-100.

10. Dickel H, Kreft B, Kuss O, Worm M. Increased sensitivity of patch testing by standardized tape stripping beforehand: A multicentre diagnostic accuracy study. Contact Dermatitis. 2010;62:294-302.

11. Davis MD, Hylwa SA, Allen EM. Basics of patch testing for allergic contact dermatitis. Semin Cutan Med Surg. 2013;32:158-68.

12. Bruynzeel DP, Andersen KE, Camarasa JG, Lachapelle JM, Menné T, White IR. The European standard series. European Environmental and Contact Dermatitis Research Group (EECDRG). Contact Dermatitis. 1995;33:145-8.

13. Thyssen JP, Johansen JD, Menné T. Contact allergy epidemics and their controls. Contact Dermatitis. 2007;56:185-95.

14. Wentworth AB, Yiannias J, Keeling JH, Hall MR, Camilleri MJ, Drage LA, et al. Trends in patch-test results and allergen changes in the standard series: A Mayo Clinic 5-year retrospective review (January 1, 2006, to December 31, 2010). J Am Acad Dermatol. 2014;70:269-75.

15. González S, Núñez R, Hurtado IC. Dermatitis de contacto alérgica en Medellín, junio 2000-agosto 2005 (tesis). Medellín: Universidad Pontificia Bolivariana; 2005.

16. Rivas AM, Kepa J, Gaviria ME, Núñez R. Estudio descriptivo de dermatitis de contacto por cosméticos en Medellín, Colombia. Rev Asoc Colomb Dermatol. 2011;19:262-70.

17. Duarte I, Rotter A, Lazzarini R. Frequency of occupational contact dermatitis in an ambulatory of dermatologic allergy. An Bras Dermatol. 2010;85:455-9.

18. Garg S, Thyssen JP, Uter W, Schnuch A. Nickel allergy following European Union regulation in Denmark, Germany, Italy and the U.K. Br J Dermatol. 2013;169:854-8.

19. Thyssen JP. Nickel and cobalt allergy before and after nickel regulation -evaluation of a public health intervention. Contact Dermatitis. 2011;65(Suppl.1):1-68.

20. Wattanakrai P, Rajatanavin N. Thimerosal allergy and clinical relevance in Thailand. J Med Assoc Thai. 2007;90:1775-9.

21. Scheman A, Rakowski EM, Chou V, Chhatriwala A. Balsam of Peru: Past and future. Dermatitis. 2013;24:153-60.

22. Uter W, Pfahlberg A, Gefeller O, Geier J, Schnuch A. Risk factors for contact allergy to nickel -results of a multifactorial analysis. Contact Dermatitis. 2003;48:33-8.
23. Zug KA, McGinley-Smith D, Warshaw EM, Taylor IS. Contact allergy in children referred for patch testing. Arch Dermatol. 2008;144:1329-36.

24. Kuljanac I, Kneževic E, Cvitanovic H. Patch test results in Karlovac county. Acta Dermatovenerol Croat. 2011;19:91-7.

25. Jacob SE, Yang A, Herro E, Zhang C. Contact allergens in a pediatric population association with atopic dermatitis and comparison with other North American referral centers. J Clin Aesthet Dermatol. 2010;3:29-35.

26. Molina SA, Pérez C, Garcés MA, Mejía M. Dermatitis de contacto en el personal del área de la salud. Revista CES Salud Pública. 2012;3:259-72. 